\title{
Non-cooperative code design in radar networks: a game-theoretic approach
}

\author{
Marco Piezzo ${ }^{1 *}$, Augusto Aubry ${ }^{2}$, Stefano Buzzi ${ }^{3}$, Antonio De Maio ${ }^{1}$ and Alfonso Farina ${ }^{4}$
}

\begin{abstract}
A network of radars sharing the same frequency band, and using properly coded waveforms to improve features attractive from the radar point of view is considered in this article. Non-cooperative games aimed at code design for maximization of the signal-to-interference plus noise ratio (SINR) of each active radar are presented. Code update strategies are proposed, and, resorting to the theory of potential games, the existence of Nash equilibria is analytically proven. In particular, we propose non-cooperative code update procedures for the cases in which a matched filter, a minimum integrated sidelobe level filter, and a minimum peak to sidelobe level filter are used the receiver. The case in which the received data contain a non-negligible Doppler shift is also analyzed. Experimental results confirm that the proposed procedures reach an equilibrium in few iterations, as well as that the SINR values at the equilibrium are largely superior to those in the case in which classical waveforms are used and no optimization of the radar code is performed.
\end{abstract}

Keywords: Game theory; Code design; Radar network; Interference mitigation; Nash equilibrium; Minimum peak-to sidelobe level (PSL) filter; Minimum integrated-to-sidelobe level (ISL) filter

\section{Introduction}

In the last decade, the importance of radar has grown progressively with the increasing dimension of the system: from a single colocated antenna to large sensor networks [1,2]. The concept of heterogeneous radars working together has thoroughly been studied, opening the door to the ideas of multiple-input multiple-output radar [3,4], over-the-horizon radar networks [5], and distributed aperture radar [6,7]. These three scenarios are the examples of cooperative radar networks, in the sense that every single sensor contributes to the overall detection process. Unfortunately, in many practical situations, it is not possible to design the network a priori. As such, the sensors are just simply added to the already existing network (plug and fight), and each sensor exhibits its own detection scheme. This is the case of non-cooperative radar networks [8,9]; in this scenario, it is extremely important that each additional sensor interferes as little as possible with the pre-existing elements, and, to

${ }^{*}$ Correspondence: marco.piezzo@unina.it

1 DIETI, University of Naples "Federico II", Napoli, Italy

Full list of author information is available at the end of the article this end, suitable techniques must be adopted. The usual approaches rely upon the employment of spatial and/or frequency diversity: the former resorts to forming multiple orthogonal beams, while the latter uses separated carrier frequencies to reduce interference [10,11]. Another possibility is to exploit waveform diversity [12-14]; here, the basic concept is to suitably modulate the waveform of the new sensor so as to optimize the detection capabilities of the specific sensor, but, at the same time, controlling the interference introduced into the network. Notice that this is different from the approach employed in cooperative sensor network, where one must design waveforms so as to optimize the joint performance of the system [15-17].

With regard to the optimization of radar waveforms in a non-cooperative scenario, we cite here the studies [18-21]. In [18], the design is based upon the maximization of the global signal-to-interference plus noise ratio (SINR), and classic constraints such as phase-only or finite energy are considered; in [19], instead, the problem of parameter estimation (e.g., direction of arrival) for a non-cooperative radar is analyzed. In this article, we propose a different strategy, based upon a game-theoretic

\section{是 Springer}

(c) 2013 Piezzo et al.: licensee Springer. This is an Open Access article distributed under the terms of the Creative Commons Attribution License (http://creativecommons.org/licenses/by/2.0), which permits unrestricted use, distribution, and reproduction in any medium, provided the original work is properly cited. 
approach [22]; we thus deal with the active radars as if they were players of a properly modeled game, whose set of possible strategies is made up of a certain amount of pre-fixed transmit radar codes. We design utility functions, based on the framework of potential games [23], trying to improve the SINR of the active radars through a non-cooperative game. Thus, we present several noncooperative games for radar-code optimization in a noncooperative environment, considering different types of receive filters [24] and accounting for the case of nonnegligible Doppler shifts too.

The remainder of this article is organized as follows. In Section 2, we give some background material on game theory and on potential games, which will be needed in the remaining part of the article. In Section 3, we present the considered radar network signal model and dwell on the proposed noncooperative games for radar code updating. Section 4 is devoted to the analysis of the performance of the proposed games, while, finally, Section 5 contains the conclusions.

\section{Brief preliminaries on game theory}

Formally speaking, a game $\mathcal{G}$ in its normal form can be described as the triplet $\mathcal{G}=\left[\mathcal{K},\left\{\mathcal{S}_{k}\right\},\left\{u_{k}\right\}\right]$, wherein $\mathcal{K}=\{1,2, \ldots, K\}$ is the set of players participating in the game, $u_{k}$ is the $k$ th player's utility function (depending on the players' chosen strategies), and $\mathcal{S}_{k}$ is the set of possible actions (strategies) that player $k$ can take. We will be considering one-shot games wherein players, in a round-robin fashion, update their strategies based on the strategies chosen by the other players, and aiming at their own utility function maximization. If, following such a strategy, an equilibrium is reached (or, in other words, if such an iterative procedure converges to a stable set of chosen strategies), then such an equilibrium is called Nash equilibrium (NE), whose formal definition is here given. Let

$$
\left(s_{1}, s_{2}, \ldots, s_{K}\right) \in \mathcal{S}_{1} \times \mathcal{S}_{2} \times \ldots \mathcal{S}_{K}
$$

denote a certain strategy $K$-tuple for the active players. Letting, as customary in the game theory literature, $\boldsymbol{s}_{-k}$ denote the $(K-1)$-dimensional vector whose entries are the strategies of all the players except the $k$ th, the point $\left(s_{1}, s_{2}, \ldots, s_{K}\right)=\left(s_{k}, s_{-k}\right)$ is an NE if, for any player $k$, we have

$$
u_{k}\left(s_{k}, s_{-k}\right) \geq u_{k}\left(s_{k}^{*}, s_{-k}\right),
$$

$\forall s_{k}^{*} \neq s_{k}$. Otherwise stated, at an NE, no user can unilaterally improve its own utility by taking a different strategy. A quick reading of this definition might lead to think that at NE users' utilities achieve their maximum values. Actually, this is not the case, since the existence of an NE point does not imply that no other strategy $K$-tuple exists that can lead to an improvement of the utilities of some players while not decreasing the utilities of the remaining ones. These latter strategies are usually said to be Paretooptimal [22]. Otherwise stated, at an NE, each player, provided that the other players' strategies do not change, is not interested in changing its own strategy. However, if some sort of cooperation would be available, players might agree to simultaneously switch to a different strategy $K$-tuple, so as to improve the utility of some, if not all, players, while not decreasing the utility of the remaining ones. The gap existing between the achieved utilities at the NE and those achieved in correspondence of Paretooptimal points is sometime colorfully named "the price of anarchy".

The concept of best response dynamic is also worth being introduced. Given a certain strategy profile $\left(s_{k}, \boldsymbol{s}_{-k}\right)$ for the active players, we say that a player implements a best response dynamic if he chooses as its new strategy $\widetilde{s}_{k}=\arg \max _{x} u_{k}\left(x, s_{-k}\right)$. Given this definition, it descends that the set of chosen strategies at an NE is the best response for every active player.

\subsection{Potential games}

A potential game [23] is a normal form game wherein any change in the utility enjoyed by a given player in reaction to a unilateral (i.e., assuming that the other players do not change their strategies) change of strategy by that player is reflected by a similar change in a global function, that is usually referred to as potential function. Formally speaking, letting $\mathcal{S}=\mathcal{S}_{1} \times \mathcal{S}_{2} \times \cdots \times \mathcal{S}_{K}$, a normal form game is an exact potential game if there exists a function $T: \mathcal{S} \rightarrow \mathcal{R}$, known as the exact potential function, such that

$$
u_{k}\left(s_{k}, s_{-k}\right)-u_{k}\left(\tilde{s}_{k}, s_{-k}\right)=T\left(s_{k}, s_{-k}\right)-T\left(\tilde{s}_{k}, s_{-k}\right),
$$

for any $k \in \mathcal{K}, s_{k}, \widetilde{s}_{k} \in \mathcal{S}_{k}$, and for any $\boldsymbol{s}_{-k} \in$ $\mathcal{S}_{1} \times \cdots \mathcal{S}_{k-1} \times \mathcal{S}_{k+1} \times \cdots \mathcal{S}_{K}$. Given a normal form game, a potential function subsumes the effects that any unilateral change of strategy may have on the utility enjoyed by that individual player. A moment of thought also reveals that every NE of an exact potential game must necessarily be a fixed point of the potential function, as well as that a best response dynamic in a 
potential game will converge to a NE in every game with continuous utility functions and compact strategy spaces [23].

Finally, it is also worth underlining that, if the potential function does represent a global performance measure for the considered system, potential games are an instance wherein users can serve the greater good while playing a non-cooperative game and acting selfishly.

In the following, we will be using game theory concepts to model competition among a set of radars (the players) that tune their own transmitted code in order to maximize their SINR. Potential games, that have been used in recent years to obtain resource allocation schemes in wireless communication applications (see, e.g., [25] and references therein), will be used here in a radar scenario to come up with procedures convergent to an NE.

\section{Problem formulation and code updating procedure}

We consider a network of $L$ non-cooperative monostatic radar systems, where each sensor transmits a coded pulse composed of $N$ sub-pulses. The signal backscattered toward the $l$ th radar is filtered through a subpulse matched filter and then converted into digital. The vector $\boldsymbol{r}_{l}, l=1, \ldots, L$, containing the received sequence $r_{k, l}, k=1, \ldots, N$, assumed temporally aligned with the returns from the range bin of interest, can be written as $[26,27]$

$$
\begin{aligned}
\boldsymbol{r}_{l}=\alpha_{0, l} \boldsymbol{c}_{l} & +\sum_{k=-N+1, k \neq 0}^{N-1} \alpha_{k, l} \boldsymbol{J}_{k} \boldsymbol{c}_{l} \\
& +\sum_{h=1, h \neq l}^{L} \sum_{k=-N+1}^{N-1} \alpha_{k, h} \boldsymbol{J}_{k} \boldsymbol{c}_{h}+\boldsymbol{n}_{l},
\end{aligned}
$$

where $c_{l}=\left[c_{l}(1) \ldots c_{l}(N)\right]^{T}$ denotes the unit-norm $N$-dimensional modulating sequence of the $l$ th radar, $\alpha_{k, h}$ are complex parameters accounting for the radar cross section of the $k$ th range bin illuminated by the $h$ th radar ( 0 is conventionally chosen as the range bin of interest), $\boldsymbol{n}_{l}$ is the vector containing the filtered thermal noise samples at the $l$ th radar (modeled as a zero-mean complex circular white vector), the matrix

$$
\boldsymbol{J}_{k}=\boldsymbol{J}_{-k}^{T}=\left\{\begin{array}{l}
\boldsymbol{J}_{k}(j, i)=1 i-j=k \\
\boldsymbol{J}_{k}(j, i)=0 \text { otherwise }
\end{array}\right.
$$

$\left(k=0, \ldots N-1,(i, j) \in\{1, \ldots, N\}^{2}\right)$ is the $N \times N$ shift matrix, and $(\cdot)^{T}$ is the transpose operator. As to the modulating sequence $c_{l}$, we suppose that it belongs to a finite set $\Omega_{l}$ which containsall the possible sequences of length $N$ that the $l$ th radar can transmit.

It is interesting to provide an interpretation of the contributions appearing in the right-hand side of (1). Indeed, the first term represents the signal component from the range bin of interest for the $l$ th radar; the second contribution accounts for the self-induced interference, while the third addend represents the interference caused by the other radars of the network on the $l$ th one.

Now, the vector $\boldsymbol{r}_{l}$ is to suitably be processed in order to detect the possible presence of a target in the range cell of interest. We thus consider the following receiving structure: the vector $\boldsymbol{r}_{l}$ undergoes a linear transformation (projection over a suitable direction vector), and, then, its square modulus is compared with a threshold, i.e., we consider the detection rule

$$
\left|\boldsymbol{d}_{l}^{\dagger}\left(\boldsymbol{c}_{l}\right) \boldsymbol{r}_{l}\right|^{2} \stackrel{H_{1}}{\underset{H_{0}}{>}} \eta_{l}
$$

with $(\cdot)^{\dagger}$ denoting conjugate transpose, $|\cdot|$ the modulus, $\boldsymbol{d}_{l}\left(\boldsymbol{c}_{l}\right)$ an $N$-dimensional vector, function of the transmit code $c_{l}$, to be suitably designed (it could be a standard matched filter or a mismatched filter [28-30] designed to optimize some performance metrics such as the integrated sidelobe level (ISL) or the peak-to-sidelobe level (PSL)-see more details in the sequel of the article), and $\eta_{l}$ the detection threshold in the $l$ th radar. Given the detection rule (3), we can define an SINR for the $l$ th radar in the range cell of interest, $\gamma_{l}$ say, as follows ${ }^{\mathrm{a}}$

$$
\gamma_{l}=\frac{\boldsymbol{G}(l, l)\left|\boldsymbol{d}_{l}^{\dagger}\left(\boldsymbol{c}_{l}\right) \boldsymbol{c}_{l}\right|^{2}}{\boldsymbol{d}_{l}^{\dagger}\left(\boldsymbol{c}_{l}\right) \boldsymbol{d}_{l}\left(\boldsymbol{c}_{l}\right)+\sum_{h=1, h \neq l}^{L} \sum_{k=-N+1}^{N-1} \boldsymbol{G}(h, l)\left|\boldsymbol{d}_{l}^{\dagger}\left(\boldsymbol{c}_{l}\right) \boldsymbol{J}_{k} \boldsymbol{c}_{h}\right|^{2}+\boldsymbol{G}(l, l) \sum_{k=-N+1, k \neq 0}^{N-1}\left|\boldsymbol{d}_{l}^{\dagger}\left(\boldsymbol{c}_{l}\right) \boldsymbol{J}_{k} \boldsymbol{c}_{l}\right|^{2}}
$$


where the matrix $\boldsymbol{G}$ models the beam-pattern of the receive antenna.

The SINR $\gamma_{l}$ is indeed a measure of the detection capabilities of the $l$ th radar in the range cell of interest. Note that at the denominator we have the contributions from the backscattered signals transmitted from the other (interfering) radars, weighted by the antenna pattern according to their direction of arrival; it thus follows that a proper design of the receive pattern helps to increase the detection capabilities.

\subsection{Antenna beam pattern}

The design of the receive antenna beam is of primary importance, especially in the case in which multiple radars operate in the same area. This problem is a classical one, and has deeply been analyzed in past years, especially with reference to wireless communications [31], where adaptive antennas are used in conjunction with power control and smart multiple access (MA) techniques. Obviously, it also plays a primary role in radar applications, where all the transmitting systems act as reciprocal sources of interference. Since we are considering here a non-cooperative scenario, no MA or a priori coordination schemes can be applied. Similarly, since the ultimate goal of a radar is to maximize its detection capability, resorting to power control is unrealistic.

In the radar scenario, the beam pattern of the antennas is used as a means to improve the received SINR and to weaken interfering echoes. A simplified model for the beam pattern $G(\theta)$ is the one illustrated in Figure 1 where $\theta=0$ is the radar search direction; for instance, such a shape can be approximated through an $N$-element array [32]. Herein, we thus assume that the antenna gain may take two possible constant values, one for $\theta \in\left[-\theta_{\epsilon},+\theta_{\epsilon}\right]$, and one (much lower than the former) outside the above interval: the side contributions are thus all equally weighted by the side beams. The effect of the antenna pattern can be therefore simply modeled as a proper $L \times L$ gain matrix $\boldsymbol{G}$, whose $(h, l)$ th element accounts for the effects of the $h$ th radar on the $l$ th system; the coefficients for $l \neq h$ are assumed to be a proper constant. The $\boldsymbol{G}(l, l)$ elements on the principal diagonal represent the main beam gain, weighting the useful signal for the $l$ th radar.

Given the outlined system model, our actual goal now is to design a non-cooperative procedure for adapting the radar codes in order to maximize the individual detection performances.

\subsection{Matched filter}

Given Equation (4), we begin with assuming that $\boldsymbol{d}_{l}=\boldsymbol{c}_{l}$, i.e., a conventional matched filter receiver is used, and consider minimization of the denominator in (4), which is equivalent to optimizing $\gamma_{l}$ since $\left\|\boldsymbol{c}_{l}\right\|=1$ ( $\|\cdot\|$ denotes the Euclidean norm of a complex vector). We thus obtain the optimization problem

$$
\begin{array}{r}
\min _{\boldsymbol{c}_{l} \in \Omega_{l}}\left\{\boldsymbol { c } _ { l } ^ { \dagger } \left(\boldsymbol{I}+\sum_{h=1, h \neq l}^{L} \sum_{k=-N+1}^{N-1} \boldsymbol{G}(h, l) \boldsymbol{J}_{k} \boldsymbol{c}_{h} \boldsymbol{c}_{h}^{\dagger} \boldsymbol{J}_{k}^{T}\right.\right. \\
\left.\left.+\boldsymbol{G}(l, l) \sum_{k=-N+1, k \neq 0}^{N-1} \boldsymbol{J}_{k} \boldsymbol{c}_{l} \boldsymbol{c}_{l}^{\dagger} \boldsymbol{J}_{k}^{T}\right) \boldsymbol{c}_{l}\right\},
\end{array}
$$

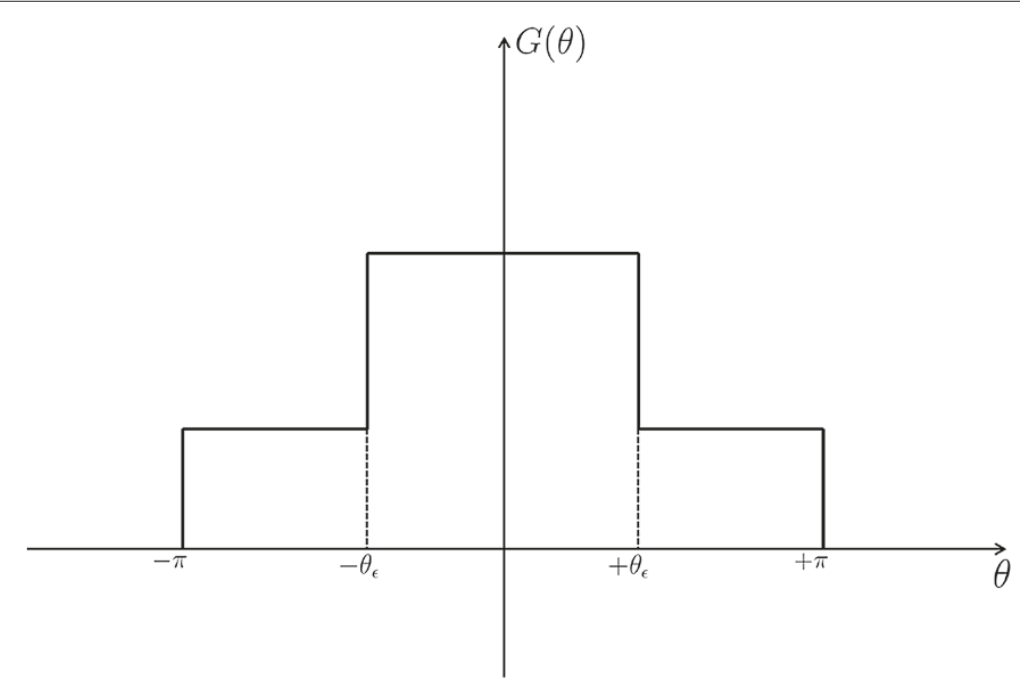

Figure 1 Antenna beam pattern. Main beam: $\theta \in\left[-\theta_{\epsilon},+\theta_{\epsilon}\right]$. Side beam: $\theta \in\left[-\pi,-\theta_{\epsilon}\right] \cup\left[+\theta_{\epsilon},+\pi\right]$. 
for $l=1, \ldots, L$. The solution for $c_{l}$ to problem (5) exists and can be found through an exhaustive optimization over the finite set $\Omega_{l}$, with an acceptable computational complexity because in practice the quoted set contains a quite small number of elements.

Unfortunately, when active radars update their own transmitted waveforms according to such a strategy, no sufficient condition has analytically been worked out for the existence of NE, and, moreover, numerical simulations have confirmed that when radars, in a round-robin fashion, update their codes according to the strategy (5), an equilibrium is not always reached. The considered game has thus no pure strategy equilibrium. One possible way to circumvent such a problem is to properly modify the utility function to be considered so that the resulting game may have an NE point. In particular, if we choose to use the tool of potential games, the trick is to define a new utility function, strictly related to (4), but whose maximization by the competing radars leads to an NE. To this end, let us consider the opposite of the sum of the denominators of $\gamma_{l}$ 's for the $L$ active radars, i.e.,

$$
\begin{gathered}
T\left(\boldsymbol{c}_{1}, \ldots, \boldsymbol{c}_{L}\right)=-\sum_{l=1}^{L} \boldsymbol{c}_{l}^{\dagger}\left(\boldsymbol{I}+\sum_{h=1, h \neq l}^{L} \sum_{k=-N+1}^{N-1}\right. \\
\boldsymbol{G}(h, l) \boldsymbol{J}_{k} \boldsymbol{c}_{h} \boldsymbol{c}_{h}^{\dagger} \boldsymbol{J}_{k}^{T}+ \\
\left.\boldsymbol{G}(l, l) \sum_{k=-N+1, k \neq 0}^{N-1} \boldsymbol{J}_{k} \boldsymbol{c}_{l} \boldsymbol{c}_{l}^{\dagger} \boldsymbol{J}_{k}^{T}\right) \boldsymbol{c}_{l} .
\end{gathered}
$$
have

Upon some straightforward algebraic manipulations, we

$$
\begin{aligned}
& T\left(\boldsymbol{c}_{1}, \ldots, \boldsymbol{c}_{L}\right)=-\boldsymbol{c}_{j}^{\dagger}\left(\boldsymbol{I}+\sum_{h=1, h \neq j}^{L} \sum_{k=-N+1}^{N-1} \boldsymbol{G}(h, j) \boldsymbol{J}_{k} \boldsymbol{c}_{h} \boldsymbol{c}_{h}^{\dagger} \boldsymbol{J}_{k}^{T}\right. \\
& \left.+\boldsymbol{G}(j, j) \sum_{k=-N+1, k \neq 0}^{N-1} \boldsymbol{J}_{k} \boldsymbol{c}_{j} \boldsymbol{c}_{j}^{\dagger} \boldsymbol{J}_{k}^{T}\right) \boldsymbol{c}_{j} \\
& -\sum_{h=1, h \neq j}^{L} \sum_{k=-N+1}^{N-1} \boldsymbol{G}(j, h) \boldsymbol{c}_{j}^{\dagger} \\
& \boldsymbol{J}_{k}^{T} \boldsymbol{c}_{h} \boldsymbol{c}_{h}^{\dagger} \boldsymbol{J}_{k} \boldsymbol{c}_{j}-T_{1}\left(\boldsymbol{c}_{1}, \ldots, \boldsymbol{c}_{j-1}, \boldsymbol{c}_{j+1}, \ldots, \boldsymbol{c}_{L}\right)
\end{aligned}
$$

where the function $T_{1}\left(\boldsymbol{c}_{1}, \ldots, \boldsymbol{c}_{j-1}, \boldsymbol{c}_{j+1}, \ldots, \boldsymbol{c}_{L}\right)$ does not depend on $\boldsymbol{c}_{j}$. In Equation (7), we have isolated the terms depending on the $j$ th radar code $c_{j}$; it thus readily follows that if we consider a game wherein the utility for the $j$ th sensor is expressed as

$$
\begin{array}{r}
u_{j}=-\boldsymbol{c}_{j}^{\dagger}\left(\boldsymbol{I}+\sum_{h=1, h \neq j}^{L} \sum_{k=-N+1}^{N-1} \boldsymbol{G}(h, j) \boldsymbol{J}_{k} \boldsymbol{c}_{h} \boldsymbol{c}_{h}^{\dagger} \boldsymbol{J}_{k}^{T}+\right. \\
\left.\boldsymbol{G}(j, j) \sum_{k=-N+1, k \neq 0}^{N-1} \boldsymbol{J}_{k} \boldsymbol{c}_{j} \boldsymbol{c}_{j}^{\dagger} \boldsymbol{J}_{k}^{T}\right) \boldsymbol{c}_{j}, \\
-\sum_{h=1, h \neq j}^{L} \sum_{k=-N+1}^{N-1} \boldsymbol{G}(j, h) \boldsymbol{c}_{j}^{\dagger} \boldsymbol{J}_{k}^{T} \boldsymbol{c}_{h} \boldsymbol{c}_{h}^{\dagger} \boldsymbol{J}_{k} \boldsymbol{c}_{j}
\end{array}
$$

we obtain an exact potential game with potential function $T(\cdot)$. Summing up, we propose the radar code update procedure reported in Algorithm 1.

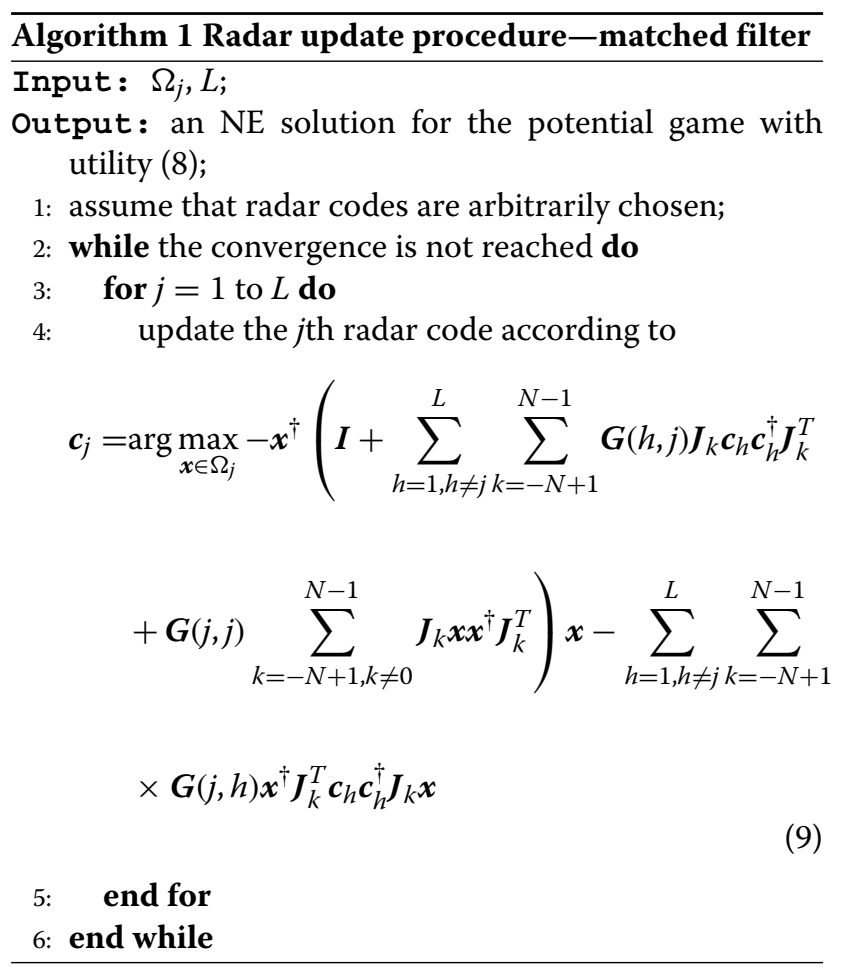

As already discussed, since at each iteration the potential function in (7) gets increased, and since it is upper bounded, it necessarily follows that the above iterative algorithm must reach a fixed point (NE). Notice however that there is in general no guarantee that such a fixed point is the global maximizer of the potential function, or just a local extremum [23].

\subsection{Minimum ISL filter}

The matched filter, considered in the previous section, is obviously the classical receiving structure used in 
detection problems. However, it does not allow to completely control the sidelobe energies, a feature that may be critical in radar applications. Indeed, this limitation may strongly affect the target detection capabilities of the radar system, especially in scenarios where multiple radars have to co-exist in the same area, thus becoming themselves the main source of reciprocal interference.

Therefore, viable alternatives to the matched filter may be sought. From this point of view, relevant metrics to be considered are the ones related to the energies in the sidelobes, which, with reference to the $l$ th radar of (1), can be modeled as $\frac{\left|\boldsymbol{d}_{l}^{\dagger}\left(\boldsymbol{c}_{l}\right) \boldsymbol{J}_{k} \boldsymbol{c}_{l}\right|^{2}}{\left|\boldsymbol{d}_{l}\left(\boldsymbol{c}_{l}\right) \boldsymbol{c}_{l}\right|^{2}}, k= \pm 1, \ldots, \pm N-1$. Specifically, if one wants to constraint the total energy underlying the sidelobes, it is possible to consider the ISL

$$
\mathrm{ISL}=\frac{\sum_{k=N+1, k \neq 0}^{N-1}\left|\boldsymbol{d}_{l}\left(\boldsymbol{c}_{l}\right)^{\dagger} \boldsymbol{J}_{k} \boldsymbol{c}_{l}\right|^{2}}{\left|\boldsymbol{d}_{l}\left(\boldsymbol{c}_{l}\right)^{\dagger} \boldsymbol{c}_{l}\right|^{2}}
$$

Indeed, designing a filter with minimum ISL is tantamount to minimizing the total energy in the range sidelobes, see, for instance, [26,33]. In particular, with reference to the $l$ th radar of model (1), the optimal ISL filter may be found as the solution to the following minimization problem:

$$
\min _{x \in \mathbb{C}^{N}} \frac{x^{\dagger} R_{l} x}{\left|x^{\dagger} c_{l}\right|^{2}}
$$

where

$$
\boldsymbol{R}_{l} \triangleq \sum_{k=N_{N}+1, k \neq 0}^{N-1} \boldsymbol{J}_{k} \boldsymbol{c}_{l} \boldsymbol{c}_{l}^{\dagger} \boldsymbol{J}_{k}^{\dagger},
$$

and $\mathbb{C}$ the complex field. It is easy to verify that a solution to (11) also solves the following constrained minimization problem

$$
\begin{aligned}
\min _{\boldsymbol{x} \in \mathbb{C}^{N}} & \boldsymbol{x}^{\dagger} \boldsymbol{R}_{l} \boldsymbol{x} \\
\text { s.t. } & \Re\left(\boldsymbol{x}^{\dagger} \boldsymbol{c}_{l}\right)=b,
\end{aligned}
$$

in the sense that $v(11)=\frac{v(12)}{b^{2}}(v(\cdot)$ stands for the optimal value of problem $(\cdot))$, for any $b>0$.

It is well known that problem (12) has a closed form solution $\boldsymbol{x}^{\star}\left(\boldsymbol{c}_{l}\right)=b \boldsymbol{Q}_{l} \boldsymbol{c}_{l}$, for any constant $b>0$, with $\boldsymbol{Q}_{l} \triangleq$ $\frac{\boldsymbol{R}_{l}^{-1}}{\boldsymbol{c}_{l}^{\dagger} \boldsymbol{R}_{l}^{-1} c_{l}}$ (indeed, it is possible to prove that $\boldsymbol{R}$ is strictly positive definite and thus invertible, provided $c_{l}(1) \neq 0$ and $c_{l}(N) \neq 0$ [34]); as a consequence, in order to solve (12), it suffices to focus on (13) with $b=1$ and $\psi=0$.
In particular, due to the direct connection between the radar code $c_{l}$ and the optimal ISL filter, as well as the energy constraint in (12), maximizing the SINR related to the signal model reported in Equation (1) is equivalent to the minimization of its denominator, i.e., the quartic form

$$
\begin{aligned}
& \min _{\boldsymbol{c}_{l} \in \Omega_{l}}\left\{\boldsymbol { c } _ { l } ^ { \dagger } \left[\boldsymbol { Q } _ { l } ^ { \dagger } \left(\boldsymbol{I}+\sum_{h=1, h \neq l}^{L} \sum_{k=-N+1}^{N-1} \boldsymbol{G}(h, l) \boldsymbol{J}_{k} \boldsymbol{c}_{h} \boldsymbol{c}_{h}^{\dagger} \boldsymbol{J}_{k}^{T}\right.\right.\right. \\
& \left.\left.\left.+\boldsymbol{G}(l, l) \sum_{k=-N+1, k \neq 0}^{N-1} \boldsymbol{J}_{k} \boldsymbol{c}_{l} \boldsymbol{c}_{l}^{\dagger} \boldsymbol{J}_{k}^{T}\right) \boldsymbol{Q}_{l}\right] \boldsymbol{c}_{l}\right\} .
\end{aligned}
$$

A solution to problem (13) can be again computed through an exhaustive search over the finite set $\Omega_{l}$; however, there is no guarantee that an equilibrium is reached as all the radars iteratively update their codes in a sequential fashion. As for the previous section, we can resort to the potential games framework to obtain an utility function for the players, such that the resulting game admits an NE. We thus consider the following potential

$$
\begin{aligned}
T\left(\boldsymbol{c}_{1}, \ldots, \boldsymbol{c}_{L}\right)= & -\sum_{l=1}^{L} \boldsymbol{c}_{l}^{\dagger}\left[\boldsymbol { Q } _ { l } ^ { \dagger } \left(\boldsymbol{I}+\sum_{h=1, h \neq l}^{L} \sum_{k=-N+1}^{N-1}\right.\right. \\
& \boldsymbol{G}(h, l) \boldsymbol{J}_{k} \boldsymbol{c}_{h} \boldsymbol{c}_{h}^{\dagger} \boldsymbol{J}_{k}^{T}+\boldsymbol{G}(l, l) \\
& \left.\left.\sum_{k=-N+1, k \neq 0}^{N-1} \boldsymbol{J}_{k} \boldsymbol{c}_{l} \boldsymbol{c}_{l}^{\dagger} \boldsymbol{J}_{k}^{T}\right) \boldsymbol{Q}_{l}\right] \boldsymbol{c}_{l},
\end{aligned}
$$

which can be rewritten as

$$
\begin{aligned}
T\left(\boldsymbol{c}_{1}, \ldots, \boldsymbol{c}_{L}\right)= & -\boldsymbol{c}_{j}^{\dagger}\left[\boldsymbol { Q } _ { j } ^ { \dagger } \left(\boldsymbol{I}+\sum_{h=1, h \neq j}^{L} \sum_{k=-N+1}^{N-1}\right.\right. \\
& \boldsymbol{G}(h, j) \boldsymbol{J}_{k} \boldsymbol{c}_{h} \boldsymbol{c}_{h}^{\dagger} \boldsymbol{J}_{k}^{T}+\boldsymbol{G}(j, j) \\
& \left.\left.\sum_{k=-N+1, k \neq 0}^{N-1} \boldsymbol{J}_{k} \boldsymbol{c}_{j} \boldsymbol{c}_{j}^{\dagger} \boldsymbol{J}_{k}^{T}\right) \boldsymbol{Q}_{j}\right] \boldsymbol{c}_{j} \\
& -\sum_{h=1, h \neq j}^{L} \sum_{k=-N+1}^{N-1} \boldsymbol{G}(j, h) \boldsymbol{c}_{j}^{\dagger} \boldsymbol{Q}_{j}^{\dagger} \boldsymbol{J}_{k}^{T} \boldsymbol{c}_{h} \boldsymbol{c}_{h}^{\dagger} \boldsymbol{J}_{k} \boldsymbol{Q}_{j} \boldsymbol{c}_{j} \\
& -T_{1}\left(\boldsymbol{c}_{1}, \ldots, \boldsymbol{c}_{j-1}, \boldsymbol{c}_{j+1}, \ldots, \boldsymbol{c}_{L}\right) .
\end{aligned}
$$


Therefore, in order to obtain an exact potential game with potential function $T(\cdot)$, we can consider the following expression for the utility of the $j$ th user:

$$
\begin{aligned}
u_{j}= & -\boldsymbol{c}_{j}^{\dagger}\left[\boldsymbol { \boldsymbol { Q } _ { j } ^ { \dagger } } \left(\boldsymbol{I}+\sum_{h=1, h \neq j}^{L} \sum_{k=-N+1}^{N-1} \boldsymbol{G}(h, j) \boldsymbol{J}_{k} \boldsymbol{c}_{h} \boldsymbol{c}_{h}^{\dagger} \boldsymbol{J}_{k}^{T}\right.\right. \\
& \left.\left.+\boldsymbol{G}(j, j) \sum_{k=-N+1, k \neq 0}^{N-1} \boldsymbol{J}_{k} \boldsymbol{c}_{j} \boldsymbol{c}_{j}^{\dagger} \boldsymbol{J}_{k}^{T}\right) \boldsymbol{Q}_{j}\right] c_{j} \\
& -\sum_{h=1, h \neq j}^{L} \sum_{k=-N+1}^{N-1} \boldsymbol{G}(j, h) c_{j}^{\dagger} \boldsymbol{Q}_{j}^{\dagger} \boldsymbol{J}_{k}^{T} \boldsymbol{c}_{h} \boldsymbol{c}_{h}^{\dagger} \boldsymbol{J}_{k} \boldsymbol{Q}_{j} \boldsymbol{c}_{j} .
\end{aligned}
$$

We summarize the steps for the radar code update procedure in the Algorithm 2.

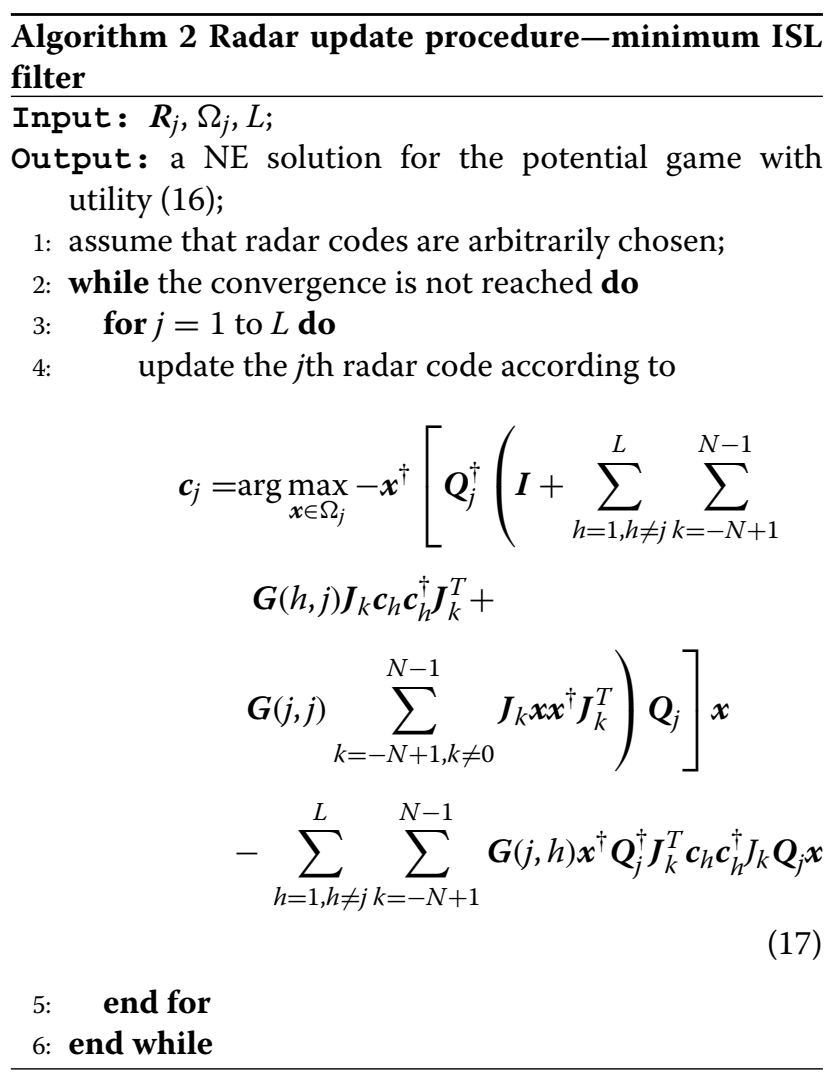

\subsection{Minimum PSL filter}

Besides the minimum ISL receive filter, another customary approach in radar applications is to constrain the level of the sidelobe peaks; the metric to be taken into account in this case is the PSL that, with reference to the $l$ th radar, can be expressed as

$$
\mathrm{PSL}=\max _{k= \pm 1, \ldots, \pm N-1} \frac{\left|\boldsymbol{d}_{l}\left(\boldsymbol{c}_{l}\right)^{\dagger} \boldsymbol{J}_{k} \boldsymbol{c}_{l}\right|^{2}}{\left|\boldsymbol{d}_{l}\left(\boldsymbol{c}_{l}\right) \boldsymbol{c}_{l}\right|^{2}} .
$$

Note that designing a filter minimizing the PSL is equivalent to cutting all the sidelobes in the filter response, and constraining the mainlobe peak to a desired level.

The computation of the minimum PSL filter is slightly more involved than the computation of the minimum ISL filter (which indeed was given in closed form), since it requires the detection of the range lobes with the highest peak level, and then their minimization; the problem can be thus formulated as the following fractional quadratic optimization problem:

$$
\min _{\boldsymbol{x} \in \mathbb{C}^{N}} \max _{k= \pm 1, \ldots, \pm N-1} \frac{\boldsymbol{x}^{\dagger} \boldsymbol{R}_{l, k} \boldsymbol{x}}{\left|\boldsymbol{x}^{\dagger} \boldsymbol{c}_{l}\right|^{2}}
$$

where

$$
\boldsymbol{R}_{l, k} \triangleq \boldsymbol{J}_{k} \boldsymbol{c}_{l} \boldsymbol{c}_{l}^{\dagger} \boldsymbol{J}_{k}^{\dagger}, \quad k= \pm 1, \ldots, \pm N-1
$$

Problem (19) can be restated into an equivalent form as

$$
\begin{array}{cl}
\min _{\boldsymbol{x} \in \mathbb{C}^{N}} \max _{k= \pm 1, \ldots, \pm N-1} & \boldsymbol{x}^{\dagger} \boldsymbol{R}_{l, k} \boldsymbol{x} \\
\text { s.t. } & \Re\left(\boldsymbol{x}^{\dagger} \boldsymbol{c}_{l}\right)=b,
\end{array}
$$

where the equivalence follows from the observation that $v((19))=\frac{v((20))}{b^{2}}$, for any $b>0$ : we can thus directly focus on (20), for $b=1$. Solving problem (20) requires the solution of a Linear Programming (LP) problem $[29,30]$ or a Second-Order Cone Programming (SOCP) [27]. Indeed, we can recast (20) as

$$
\begin{array}{rl}
\min _{t, \boldsymbol{x}} & t \\
\mathrm{s.t.} & t \geq \boldsymbol{x}^{\dagger} \boldsymbol{R}_{l, k} \boldsymbol{x}, \quad k= \pm 1, \ldots, \pm N-1, \\
& \Re\left(\boldsymbol{x}^{\dagger} \boldsymbol{c}_{l}\right)=1,
\end{array}
$$

which belongs to the class of the LP $[29,30]$ or SOCP [27] problems for the case of real or complex transmitted code sequence and optimization variable, respectively.

Obviously, an optimal solution $\boldsymbol{x}^{\star}$ for problem (21) is a function of the radar code $c_{l}$ used by the player; therefore, the finite set $\Omega_{l}$ of the possible radar sequences and the set, say $\Sigma_{l}$, of the possible optimal PSL filters are related by a one-to-one correspondence. Otherwise stated, specifying $\Omega_{l}$ also leads to specify $\Sigma_{l}$, in the sense that the set of the filters can be computed directly off-line, and populated by the possible solutions for the problem (21). 
Based on the above assumptions, the maximization of the SINR for the pair $\left(\boldsymbol{c}_{l}, \boldsymbol{d}_{l}\left(\boldsymbol{c}_{l}\right)\right)$ relative to (21), leads to the following minimization

$$
\begin{array}{r}
\min _{\boldsymbol{c}_{l} \in \Omega_{l}}\left\{\boldsymbol { d } _ { l } ( \boldsymbol { c } _ { l } ) ^ { \dagger } \left(\boldsymbol{I}+\sum_{h=1, h \neq l}^{L} \sum_{k=-N+1}^{N-1} \boldsymbol{G}(h, l) \boldsymbol{J}_{k} \boldsymbol{c}_{h} \boldsymbol{c}_{h}^{\dagger} \boldsymbol{J}_{k}^{T}\right.\right. \\
\left.\left.+\boldsymbol{G}(l, l) \sum_{k=-N+1, k \neq 0}^{N-1} \boldsymbol{J}_{k} \boldsymbol{c}_{l} \boldsymbol{c}_{l}^{\dagger} \boldsymbol{J}_{k}^{T}\right) \boldsymbol{d}_{l}\left(\boldsymbol{c}_{l}\right)\right\} .
\end{array}
$$

where, for each transmitted sequence $c_{l} \in \Omega_{l}$, it is necessary to consider the corresponding filter $\boldsymbol{d}_{l}\left(\boldsymbol{c}_{l}\right) \in \Sigma_{l}$. Again, for the purpose of correctly modeling the game among the $L$ users, let us define the following potential:

$$
\begin{gathered}
T\left(\boldsymbol{c}_{1}, \ldots, \boldsymbol{c}_{L}\right)=-\sum_{l=1}^{L} \boldsymbol{d}_{l}\left(\boldsymbol{c}_{l}\right)^{\dagger}\left(\boldsymbol{I}+\sum_{h=1, h \neq l}^{L} \sum_{k=-N+1}^{N-1}\right. \\
\boldsymbol{G}(h, l) \boldsymbol{J}_{k} \boldsymbol{c}_{h} \boldsymbol{c}_{h}^{\dagger} \boldsymbol{J}_{k}^{T}+\boldsymbol{G}(l, l) \\
\left.\sum_{k=-N+1, k \neq 0}^{N-1} \boldsymbol{J}_{k} \boldsymbol{c}_{l} \boldsymbol{c}_{l}^{\dagger} \boldsymbol{J}_{k}^{T}\right) \boldsymbol{d}_{l}\left(\boldsymbol{c}_{l}\right),
\end{gathered}
$$

where we assume that the correspondence between filters and transmitted sequences has already been defined. Specifically, we may resort to Table 1 , that can be looked upon during the update procedure. After some algebraic transformations, we obtain

$$
\begin{aligned}
T\left(\boldsymbol{c}_{1}, \ldots, \boldsymbol{c}_{L}\right)= & -\boldsymbol{d}_{j}\left(\boldsymbol{c}_{j}\right)^{\dagger}\left(\boldsymbol{I}+\sum_{h=1, h \neq j}^{L} \sum_{k=-N+1}^{N-1}\right. \\
& \boldsymbol{G}(h, j) \boldsymbol{J}_{k} \boldsymbol{c}_{h} \boldsymbol{c}_{h}^{\dagger} \boldsymbol{J}_{k}^{T}+\boldsymbol{G}(j, j) \\
& \left.\sum_{k=-N+1, k \neq 0}^{N-1} \boldsymbol{J}_{k} \boldsymbol{c}_{j} \boldsymbol{c}_{j}^{\dagger} \boldsymbol{J}_{k}^{T}\right) \boldsymbol{d}_{j}\left(\boldsymbol{c}_{j}\right)-\sum_{h=1, h \neq j}^{L} \\
& \sum_{k=-N+1}^{N-1} \boldsymbol{G}(j, h) \boldsymbol{d}_{h}\left(\boldsymbol{c}_{h}\right)^{\dagger} \boldsymbol{J}_{k}^{T} \boldsymbol{c}_{j} \boldsymbol{c}_{j}^{\dagger} \boldsymbol{J}_{k} \boldsymbol{d}_{h}\left(\boldsymbol{c}_{h}\right) \\
& -T_{1}\left(\boldsymbol{c}_{1}, \ldots, \boldsymbol{c}_{j-1}, c_{j+1}, \ldots, \boldsymbol{c}_{L}\right) .
\end{aligned}
$$

Given the above potential function, it is possible to define the utility for the $j$ th user as

$$
\begin{aligned}
u_{j}= & -\boldsymbol{d}_{j}\left(\boldsymbol{c}_{j}\right)^{\dagger}\left(\boldsymbol{I}+\sum_{h=1, h \neq j}^{L} \sum_{k=-N+1}^{N-1} \boldsymbol{G}(h, j) \boldsymbol{J}_{k} \boldsymbol{c}_{h} \boldsymbol{c}_{h}^{\dagger} \boldsymbol{J}_{k}^{T}\right. \\
& \left.+\boldsymbol{G}(j, j) \sum_{k=-N+1, k \neq 0}^{N-1} \boldsymbol{J}_{k} \boldsymbol{c}_{j} \boldsymbol{c}_{j}^{\dagger} \boldsymbol{J}_{k}^{T}\right) \boldsymbol{d}_{j}\left(\boldsymbol{c}_{j}\right) \\
& -\sum_{h=1, h \neq j}^{L} \sum_{k=-N+1}^{N-1} \boldsymbol{G}(j, h) \boldsymbol{d}_{h}\left(\boldsymbol{c}_{h}\right)^{\dagger} \boldsymbol{J}_{k}^{T} \boldsymbol{c}_{s} \boldsymbol{c}_{j}^{\dagger} \boldsymbol{J}_{k} \boldsymbol{d}_{h}\left(\boldsymbol{c}_{h}\right),
\end{aligned}
$$

whose iterative maximization by the active radars leads to a new potential game admitting NE points. Algorithm 3 summarizes the radar code update iterations for the case at hand. ${ }^{\mathrm{c}}$

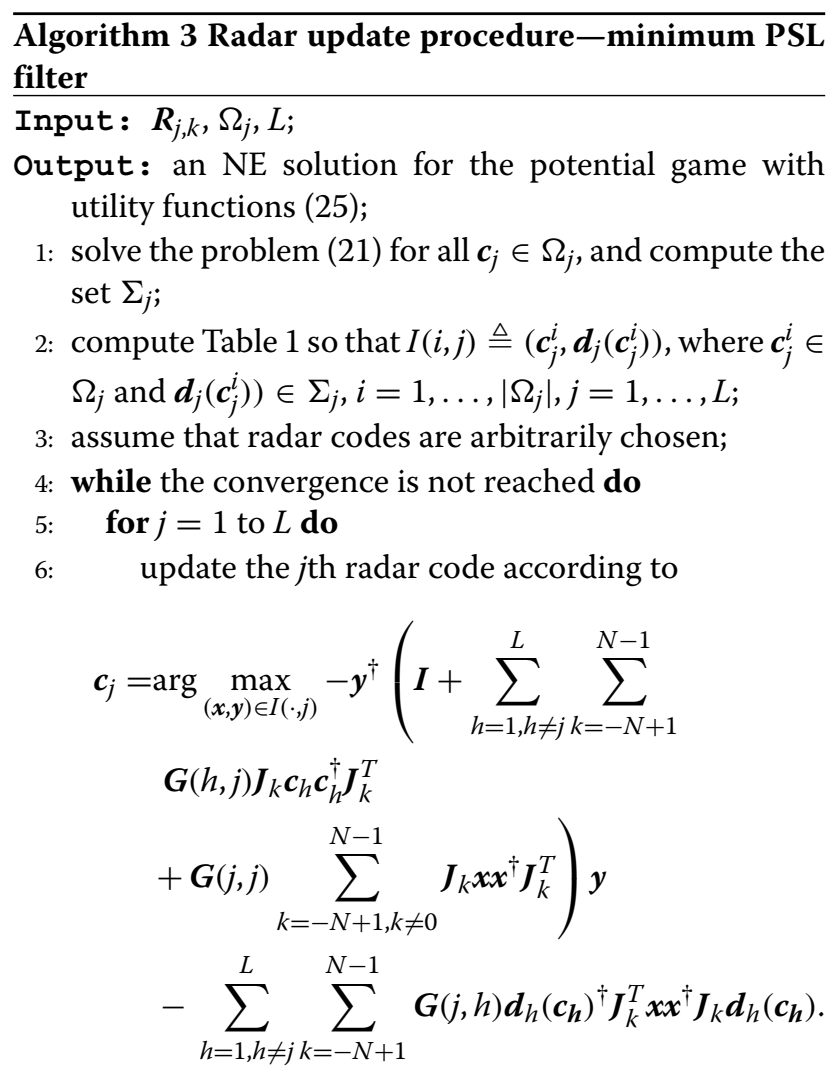

7: $\quad$ end for

8: end while

\subsection{Non-negligible Doppler shift}

So far, we have implicitly assumed that the received signal is affected by either null or negligible Doppler shift. However, it is well known that if the targets illuminated 
Table 1 Classes of phase-codes $\phi$

\begin{tabular}{llll}
\hline Parameters & $\boldsymbol{\phi}_{\boldsymbol{I}}$ & Parameters & $\boldsymbol{\phi}_{\boldsymbol{I}}$ \\
\hline$r=15$ & Golomb-Zhang & - & Palindronic P4 \\
- & MPS (Minimum Peak Sidelobe) & $r=3$ & Chu \\
- & Zadoff-Chu & $r=13$ & Golomb-Zhang \\
$r=5, q=10$ & Zadoff & $r=17$ & Chu \\
$r=27, q=8$ & Zadoff & $r=3, q=16$ & Zadoff \\
- & P3 & $r=21, q=0$ & Zadoff \\
$r=3$ & Golomb-Zhang & - & - \\
\hline
\end{tabular}

Classes of phase-codes $\phi$; length $N=16 ;\{r, q\}$ design parameters [35].

by the network of radars rapidly change their position with unknown velocity and directions, then it is necessary to account for the effect (no more negligible) of the Doppler frequency shifts. To this end, we follow the same approach as in [27], extending it to the considered noncooperative scenario. Specifically, let us assume that $\omega_{l}=$ $\left[\omega_{-N+1, l}, \ldots, \omega_{N-1, l}\right]$ is the Doppler shifts vector for the $l$ th radar, with $l=1, \ldots, L$. Moreover, let

$$
c_{l}\left(\omega_{k, l}\right)=\left[c_{l}(1) e^{j \omega_{k, l}} \ldots c_{l}(N) e^{j N \omega_{k, l}}\right]^{T}
$$

be the related Doppler shifted code sequence. The data model (1) can be thus modified as follows:

$$
\begin{aligned}
\boldsymbol{r}_{l}=\alpha_{0, l} \boldsymbol{c}_{l}\left(\omega_{0, l}\right) & +\sum_{k=-N+1, k \neq 0}^{N-1} \alpha_{k, l} \boldsymbol{J}_{k} \boldsymbol{c}_{l}\left(\omega_{k, l}\right) \\
& +\sum_{h=1, h \neq l}^{L} \sum_{k=-N+1}^{N-1} \alpha_{k, h} \boldsymbol{J}_{k} \boldsymbol{c}_{h}\left(\omega_{k, h}\right)+\boldsymbol{n}_{l},
\end{aligned}
$$

wherein $\omega_{0, l}$ is the Doppler shift associated to the range bin of interest. Now, should such a Doppler shift be known at the receiver, the following detection rule should be considered:

$$
\left|\boldsymbol{d}_{l}^{\dagger}\left(\boldsymbol{c}_{l}\left(\omega_{0, l}\right)\right) \boldsymbol{r}_{l}\right|^{2} \underset{H_{0}}{\stackrel{H_{1}}{\gtrless}} \eta_{l},
$$

with $\boldsymbol{d}_{l}\left(\boldsymbol{c}_{l}\left(\omega_{0, l}\right)\right)$ the $N$-dimensional detection vector, function of the (known) Doppler shifted code $\boldsymbol{c}_{l}\left(\omega_{0, l}\right)$. Given Equation (29), the SINR equation (4) may easily be reformulated as follows:
In practical radar applications, however, the target Doppler shift is usually unknown, and the available knowledge is limited to the range $\left[\omega_{0}, \omega_{1}\right]$ of variability of the Doppler frequencies. The customary approach thus relies on a quantization of the said interval with a preassigned resolution $(\Delta \omega)$ (a typical value of $(\Delta \omega)$ is $\pi /(10 N)$ [27]) and, at the reception side, a bank of detection vectors, each one keyed to one of the quantized Doppler frequencies, is considered, followed by a maximum selector. Otherwise stated, denoting by $\omega(1), \omega(2), \ldots, \omega(P)$ the $P$ sample frequencies obtained by sampling with step $(\Delta \omega)$ the interval $\left[\omega_{0}, \omega_{1}\right]$, the detection rule is actually expressed as

$$
\max _{i \in\{1,2, \ldots, P\}}\left|\boldsymbol{d}_{l}^{\dagger}\left(\boldsymbol{c}_{l}(\omega(i))\right) \boldsymbol{r}_{l}\right|^{2} \stackrel{H_{1}}{\underset{H_{0}}{<}} \eta_{l} .
$$

Now, in order to come up with a code update procedure, we should still focus on the minimization of the denominator of Equation (30); note however that such a denominator depends on the Doppler shifts $\left\{\omega_{k, l}\right\}$, with $l=$ $1, \ldots, L$ and $k=-N+1, \ldots, N-1$. In order to circumvent this drawback, a suitable technique is to consider the statistical expectation of the denominator of (30), averaged with respect to the set of Doppler shifts. Since in practice the detection vectors are considered only for a finite number of Doppler frequencies, in performing the average we model the detection vector as taking value in the discrete set $\left\{\boldsymbol{d}_{l}^{\dagger}\left(\boldsymbol{c}_{l}(\omega(1))\right), \boldsymbol{d}_{l}^{\dagger}\left(\boldsymbol{c}_{l}(\omega(2))\right), \ldots, \boldsymbol{d}_{l}^{\dagger}\left(\boldsymbol{c}_{l}(\omega(P))\right)\right\}$, while the frequencies inside the curly brackets in the denominator of Equation (30) are assumed to be continuous and uniform random variates taking value in the set

$$
\gamma_{l}=\frac{\boldsymbol{G}(l, l)\left|\boldsymbol{d}_{l}^{\dagger}\left(\boldsymbol{c}_{l}\left(\omega_{0, l}\right)\right) \boldsymbol{c}_{l}\left(\omega_{0, l}\right)\right|^{2}}{\boldsymbol{d}_{l}^{\dagger}\left(\boldsymbol{c}_{l}\left(\omega_{0, l}\right)\right)\left(\begin{array}{l}
\boldsymbol{I}+\sum_{h=1, h \neq l}^{L} \sum_{k=-N+1}^{N-1} \boldsymbol{G}(h, l) \boldsymbol{J}_{k} \boldsymbol{c}_{h}\left(\omega_{k, h}\right) \boldsymbol{c}_{h}^{\dagger}\left(\omega_{k, h}\right) \boldsymbol{J}_{k}^{T}+ \\
+\boldsymbol{G}(l, l) \sum_{k=-N+1, k \neq 0}^{N-1} \boldsymbol{J}_{k} \boldsymbol{c}_{l}\left(\omega_{k, l}\right) \boldsymbol{c}_{l}^{\dagger}\left(\omega_{k, l}\right) \boldsymbol{J}_{k}^{T}
\end{array}\right) \boldsymbol{d}_{l}\left(\boldsymbol{c}_{l}\left(\omega_{0, l}\right)\right)} .
$$


$\left[\omega_{0}, \omega_{1}\right]$. For the case in which a matched filter is used at the receiver, the presence of non-negligible Doppler shifts thus leads to consider, in place of Equation (7),the following potential function:

$$
\begin{aligned}
& T\left(\boldsymbol{c}_{1}, \ldots, \boldsymbol{c}_{L}\right)=-\sum_{l=1}^{L} \sum_{i=1}^{P}\left\{\boldsymbol { c } _ { l } ^ { \dagger } ( \omega ( i ) ) \left(\boldsymbol{I}+\sum_{h=1, h \neq l}^{L} \sum_{k=-N+1}^{N-1}\right.\right. \\
& \boldsymbol{G}(h, l) \boldsymbol{J}_{k} \overline{\boldsymbol{c}_{h}\left(\omega_{k, h}\right) \boldsymbol{c}_{h}^{\dagger}\left(\omega_{k, h}\right)} \boldsymbol{J}_{k}^{T} \\
& \left.\left.+\boldsymbol{G}(l, l) \sum_{k=-N+1, k \neq 0}^{N-1} \boldsymbol{J}_{k} \overline{\boldsymbol{c}_{l}\left(\omega_{k, l}\right) \boldsymbol{c}_{l}^{\dagger}\left(\omega_{k, l}\right)} \boldsymbol{J}_{k}^{T}\right) \boldsymbol{c}_{l}(\omega(i))\right\} \text {, }
\end{aligned}
$$

wherein the overline $\overline{(\cdot)}$ denotes statistical expectation with respect to the Doppler shifts; note that, upon letting $\boldsymbol{F}_{l}=\overline{\boldsymbol{c}_{l}\left(\omega_{k, l}\right) \boldsymbol{c}_{l}^{\dagger}\left(\omega_{k, l}\right)}$, it is easily shown that the $(n, m)$ th entry of $\boldsymbol{F}_{l}$, say $\boldsymbol{F}_{l}(n, m)$, is expressed as

$$
\begin{aligned}
& \boldsymbol{F}_{l}(n, m)=\frac{c_{l}(n) c_{l}^{\dagger}(m)}{\omega_{1}-\omega_{0}} \int_{\omega_{0}}^{\omega_{1}} e^{j(n-m) \omega} d \omega= \\
& \begin{cases}\left|c_{l}(n)\right|^{2}, & n=m, \\
\frac{c_{l}(n) c_{l}^{\dagger}(m)\left[e^{j(n-m) \omega_{1}}-e^{j(n-m) \omega_{0}}\right]}{j(n-m)\left[\omega_{1}-\omega_{0}\right]}, & n \neq m .\end{cases}
\end{aligned}
$$

Now, given the potential function (32), a noncooperative game can be obtained, similar to the case of negligible Doppler shift, by isolating the terms depending on a given code, say the $j$ th. The utility function for the $j$ th radar is thus written as

$$
\begin{aligned}
u_{j}= & -\sum_{i=1}^{P}\left\{\boldsymbol { c } _ { j } ^ { \dagger } ( \omega ( i ) ) \left(\boldsymbol{I}+\sum_{h=1, h \neq j}^{L} \sum_{k=-N+1}^{N-1} \boldsymbol{G}(h, j) \boldsymbol{J}_{k} \boldsymbol{F}_{h} \boldsymbol{J}_{k}^{T}\right.\right. \\
& \left.\left.+\boldsymbol{G}(j, j)+\sum_{k=-N+1, k \neq 0}^{N-1} \boldsymbol{J}_{k} \boldsymbol{F}_{j}\left(\boldsymbol{c}_{j}\right) \boldsymbol{J}_{k}^{T}\right) \boldsymbol{c}_{j}(\omega(i))\right\} \\
& -\sum_{l=1, l \neq j}^{L} \sum_{i=1}^{P}\left\{\boldsymbol{c}_{l}^{\dagger}(\omega(i))\left(\sum_{k=-N+1}^{N-1} \boldsymbol{G}(j, l) \boldsymbol{J}_{k} \boldsymbol{F}_{j}\left(\boldsymbol{c}_{j}\right) \boldsymbol{J}_{k}^{T}\right) \boldsymbol{c}_{l}(\omega(i))\right\} .
\end{aligned}
$$

In writing the above equation, we have made explicit the functional dependence of the matrix $\boldsymbol{F}_{j}$ on the code $c_{j}$, which has to properly be accounted for in the utility maximization. Summing up, for non-negligible Doppler shifts and matched filter reception, each radar should update its code to maximize the utility in (34), and the detection rule to be considered should be the one reported in Equation (31).

Similar considerations can be done for the cases in which a minimum ISL or PSL filters are used. For the sake of brevity, however, we avoid providing more details on this, since it would not add conceptual value to this study.

\section{Performance analysis}

In this section, we assess the performance of the proposed non-cooperative waveform design techniques; to this end, we test the outlined algorithms in two distinct operational scenarios, where the difference is mainly in the number of involved radars, as well as their receive antenna pattern characterization. Precisely, we consider the following two games $\mathcal{G}_{i}=\left\{\mathcal{L}_{i}, \Omega_{l},\left\{u_{l}\right\}\right\}$, for $i=\{1,2\}$ where

- $\mathcal{L}_{1}=\{1,2,3,4\}$ is the set of 4 players (i.e., the set of four radars actually transmitting), while $\mathcal{L}_{2}=\{1,2,3,4,5,6\}$ is the set of six players (i.e., the set of six radars actually transmitting);

- $\Omega_{l}$ is a set of cardinality $M=653$ which contains the sequences of length $N=16$ available to the 1 th player. The same set is considered for each radar, i.e., $\Omega_{l}$ is actually independent of the index $l$ (and indeed we will be denoting it by $\Omega$ in the following). The full details on the sequences of the set $\Omega$ are reported in Appendix.

- $\left\{u_{l}\right\}$ represents the utility function for the 1 th player, as defined in the discussed Algorithms 1, 2, and 3, for $l=1, \ldots, 4$ or $l=1, \ldots, 6$, respectively for the first and the second games;

- $\boldsymbol{G}$ is the $L_{i} \times L_{i}$ matrix describing the antenna gain pattern of the $L_{i}$ players, for $i=\{1,2\}$. We consider a general scenario wherein each radar may have its own antenna beam pattern, but we normalize, without loss of generality, to $0 \mathrm{~dB}$ the maximum gain of each antenna. Indeed, we consider the following pattern models for the games $\mathcal{G}_{1}$ and $\mathcal{G}_{2}$ :

$$
\begin{aligned}
& \boldsymbol{G}_{\mathcal{G}_{1 \mathrm{~dB}}}=\left[\begin{array}{cccc}
0 & -30 & -19 & -20 \\
-20 & 0 & -19 & -20 \\
-20 & -30 & 0 & -20 \\
-20 & -30 & -19 & 0
\end{array}\right], \\
& \boldsymbol{G}_{\mathcal{G}_{2 \mathrm{~dB}}}=\left[\begin{array}{cccccc}
0 & -30 & -19 & -20 & -15 & -23 \\
-20 & 0 & -19 & -20 & -15 & -23 \\
-20 & -30 & 0 & -20 & -15 & -23 \\
-20 & -30 & -19 & 0 & -15 & -23 \\
-20 & -30 & -19 & -20 & 0 & -23 \\
-20 & -30 & -19 & -20 & -15 & 0
\end{array}\right] \text {, }
\end{aligned}
$$

respectively. ${ }^{\mathrm{d}}$

With reference to the simulation setup of Figures 2a, 3a, and $4 \mathrm{a}$, we choose four transmit sequences from $\Omega$ and consider them as the initial strategies for $\mathcal{G}_{1}$. Moreover, 


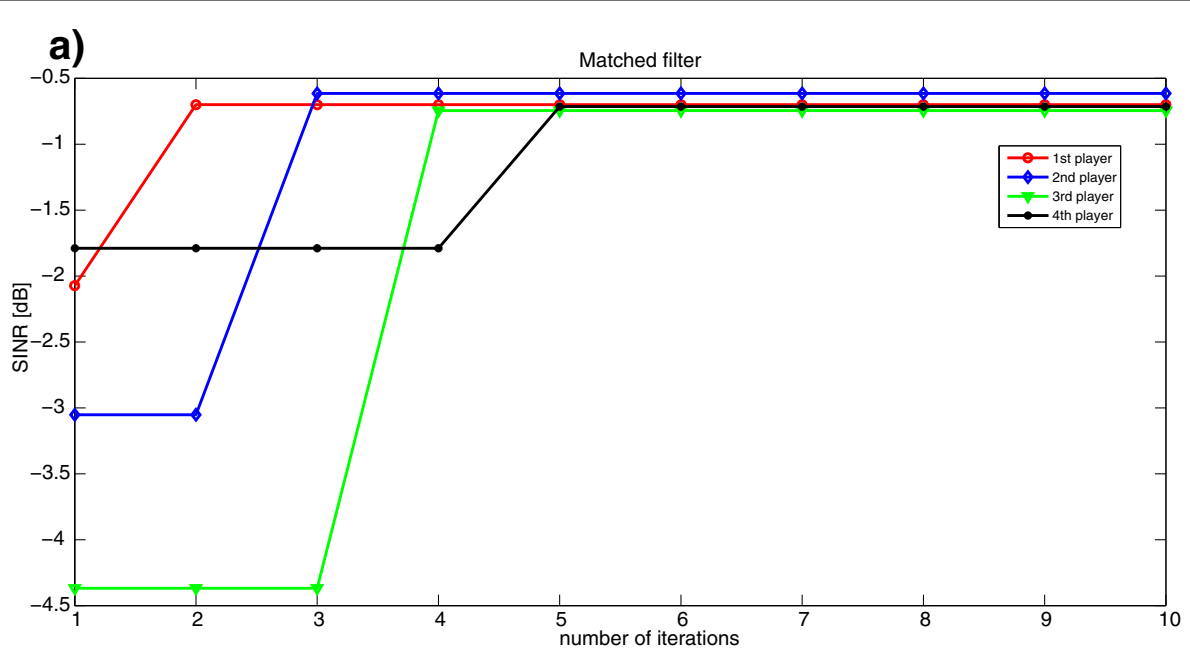

b)

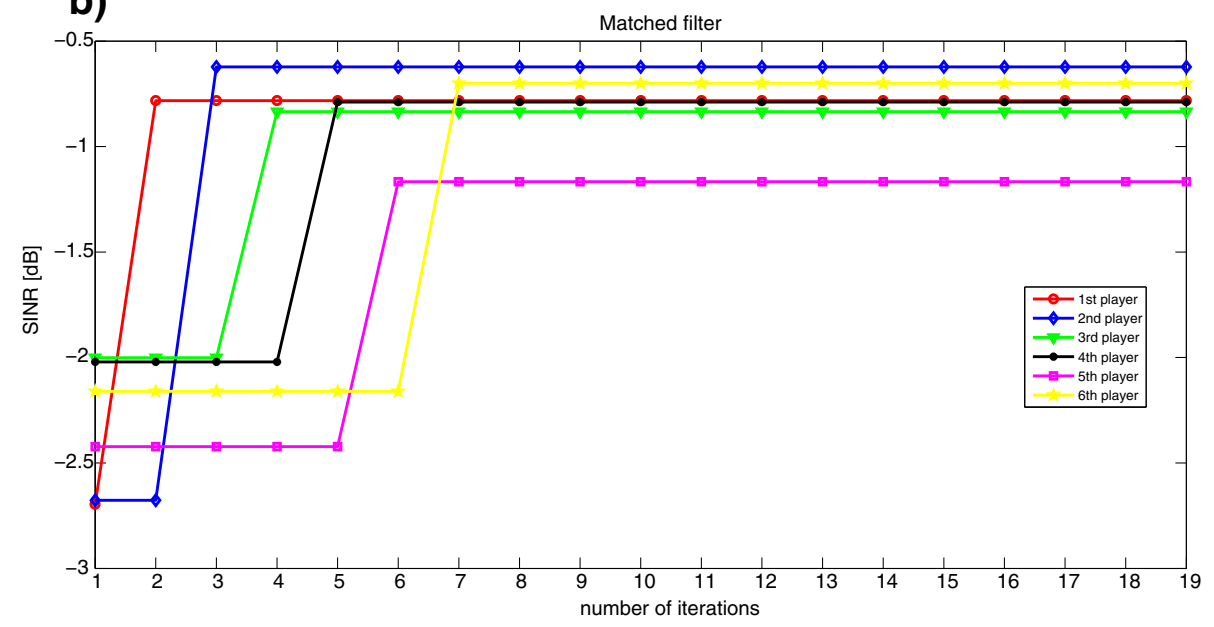

Figure 2 SINR versus the number of iterations, for a set of (a) $L=4$ players, Algorithm 1, (b) $L=6$ players, Algorithm 1 .

for the corresponding figures of $\mathcal{G}_{2}$ (Figures $2 \mathrm{~b}, 3 \mathrm{~b}$, and $4 \mathrm{~b})$, we add two more codes, still selected from $\Omega$, to the four aforementioned initial strategies. The analysis is conducted in terms of SINR $\gamma_{l}$ which each player is able to obtain through the non-cooperative code design, focusing on the performance provided by the three algorithms. The average SINR among the transmitting radars at the equilibrium, for all the players, as their number increases, is also plotted. The results emphasize how, as the number of interferers increases, the games actually are able to reduce the consequential loss of performances with respect to the case in which no code optimization procedure is performed.

In Figure 2a,b, we plot the SINR of each player versus the number of iterations required by Algorithm 1 to converge to an NE, for the games $\mathcal{G}_{1}$ and $\mathcal{G}_{2}$, respectively; these plots show the impact of the chosen code (strategy) on the SINR of the set of players, as they pick up different codes from the set $\Omega$. Note that the starting codes (strategies) do not provide satisfactory values of $\gamma_{l}$ for all the set of players; indeed, in both the games the majority of the sensors experiment quite a low level of SINR, with the exception of the first and the last players. The curves highlight that, as the players change their transmitting codes according to Algorithm 1, the SINR of each player converges to a fixed value: after a certain amount of iterations, the iterative algorithm thus reaches a fixed code (strategy). In particular, both the sets of players share an average increase in their respective performances, quantifiable in about $2.10 \mathrm{~dB}$ for the first game and $1.51 \mathrm{~dB}$ for the second game, and no particular loss is observed due to the growth of the number of transmitting radars. Moreover, convergence is reached after a few iterations.

In Figure $3 \mathrm{a}, \mathrm{b}$, the same analysis is conducted for Algorithm 2. Again, the starting strategy seems to be quite disadvantageous for both the sets of active radars, and in particular for the second game (specifically, we experience 

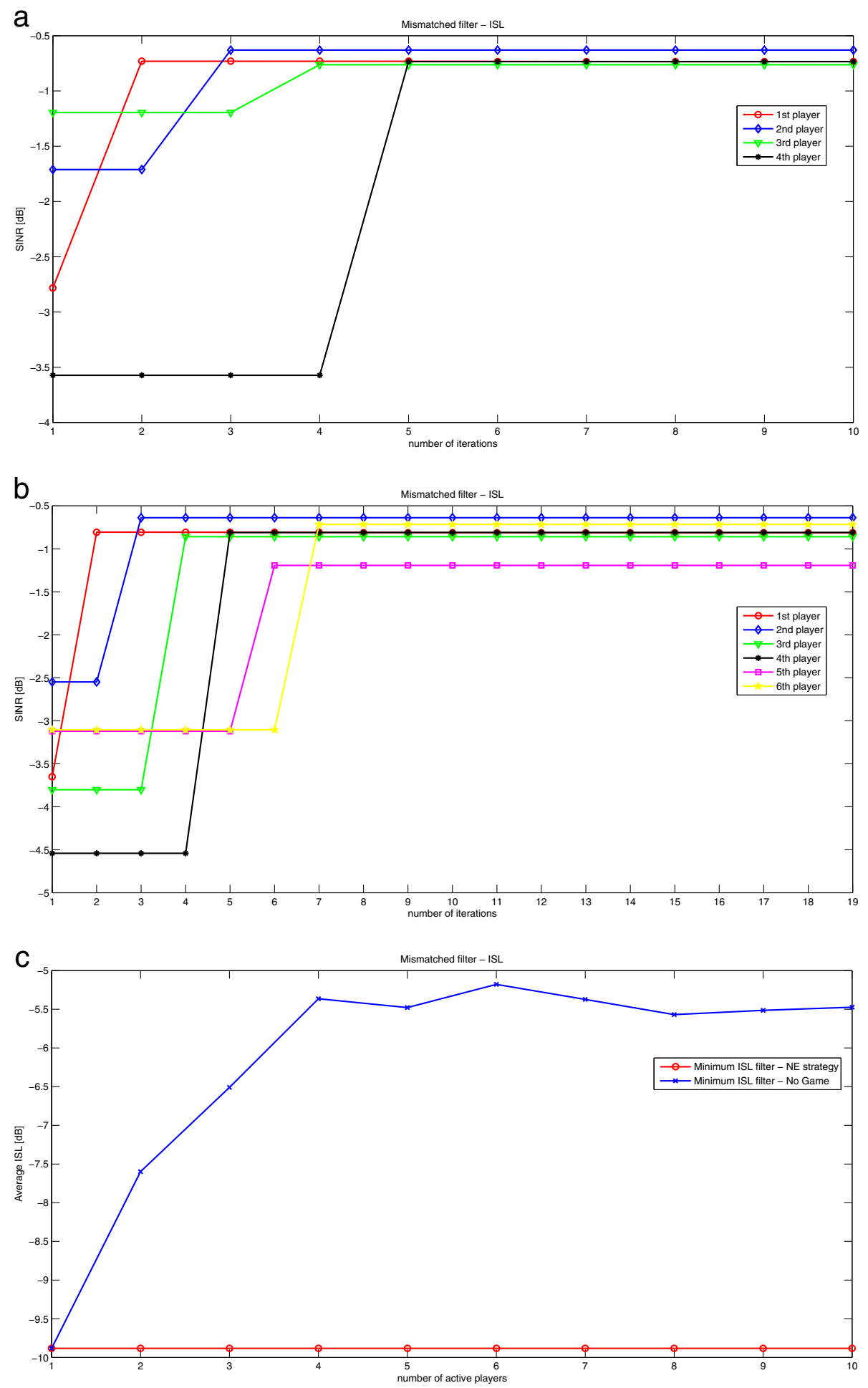

Figure 3 SINR versus the number of iterations, for a set of (a) $L=4$ players, (b) $L=6$ players, Algorithm 2. (c) Average ISL versus the number of active players, Algorithm 2. ISL at the NE points (solid-circle red line); ISL with a random choice (solid-cross blue line). 

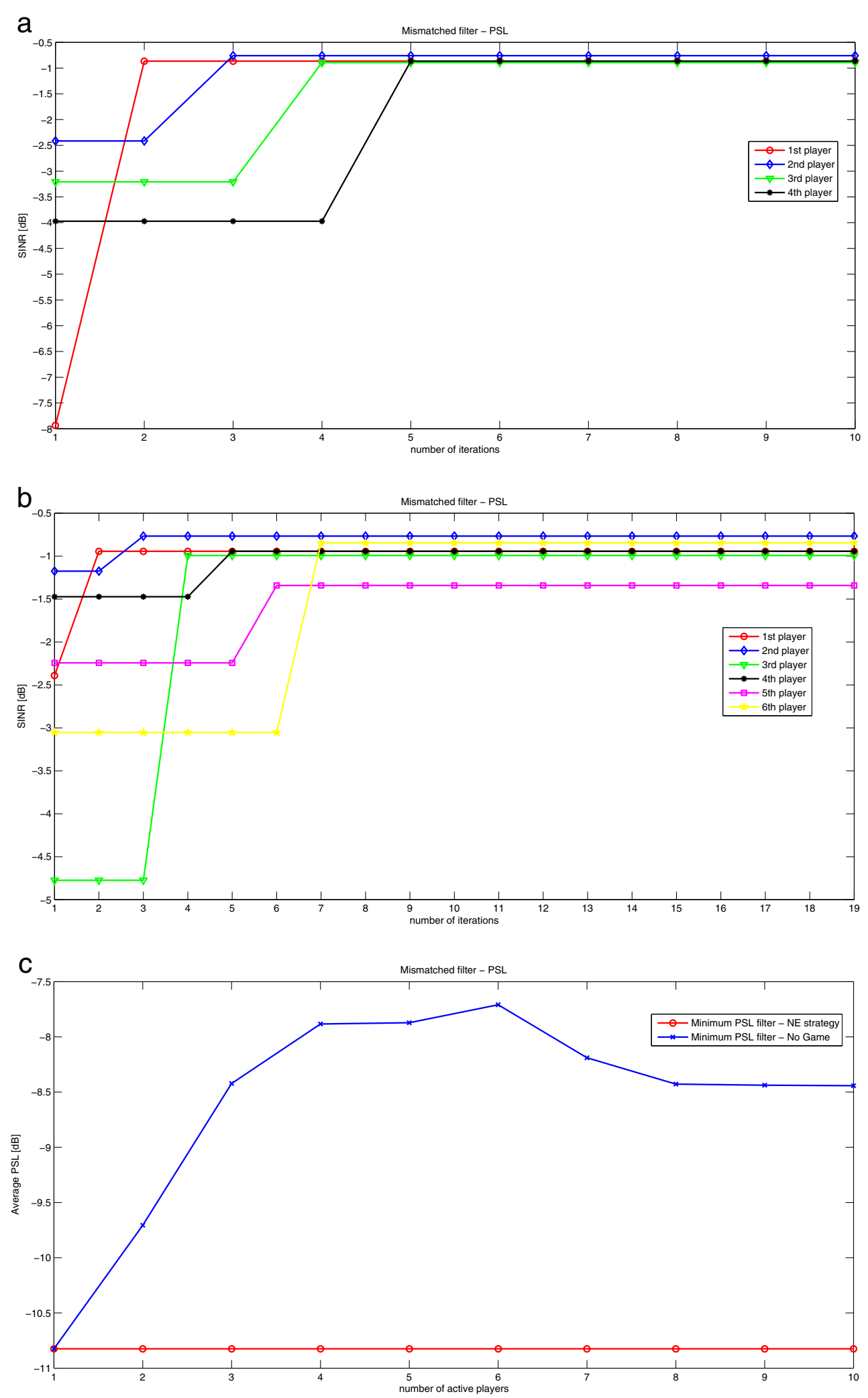

Figure 4 SINR versus the number of iterations, for a set of $L=\mathbf{4}$ (a) and $L=\mathbf{6}$ (b) players, Algorithm 3. (c) Average PSL versus the number of active players, Algorithm 3. PSL at the NE points (solid-circle red line); PSL with a random choice (solid-cross blue line). 
unpleasant performances in the cases of radars 1 and 4, with reference to the first game, and radars 3-4 for the second game). Resorting to the coding procedure of Algorithm 2, however, all the radars increase the respective performances; in particular, we observe an average increase, in the achieved SINR values, of $1.56 \mathrm{~dB}$ for game $\mathcal{G}_{1}$ and $2.66 \mathrm{~dB}$ for game $\mathcal{G}_{2}$. The analysis also shows a gain in terms of ISL values, due to the game approach. Specifically, in Figure 3c, we provide a comparison between the average ISL, with respect to the increasing number of active radars (for the case at hand, we assume a maximum of ten radars), obtained with the Algorithm 2 and the no-game strategy, respectively. In the setup of this simulation, random initial strategies have been selected for the radars and the results have been averaged over 25 independent trials. The plots highlight that the no-game approach is very sensitive to the number of sensors composing the network; in fact increasing values of ISL can be observed when the number of active radars increases. On the contrary, the updating procedure of Algorithm 2 is capable of ensuring a quite flat ISL behavior.

In Figure 4a,b, we focus on the performance of Algorithm 3, and similar comments as for the previous two algorithms can be made. The average increase, in terms of SINR, can be quantified in $3.60 \mathrm{~dB}$ for the first game, and $1.19 \mathrm{~dB}$ for the second one.

In Figure 4c, we consider the average PSL versus the number of active radars, for both the no-game approach and the non-cooperative game technique of Algorithm 3. The same simulation conditions as in Figure 3c have been considered concerning the initial choice. Notice that the average PSL for the no-game approach appears quite unpleasant, as worse and worse PSL values are obtained increasing the number of active sensors. On the contrary, Algorithm 3 seems quite robust in terms of PSL with respect to the number of active radars.

Finally, in Figure 5, we analyze the average SINR among all the radars, at the NE, versus the number of active radars in the network, with respect to Algorithms 1, 2 , and 3; in particular, for the latter two algorithms, the curves refer to the SINR values for the NE points of Figures $3 \mathrm{c}$ and $4 \mathrm{c}$ (as such random initial strategies have been considered for the radars operate according to a no-game approach and the results are averaged over 25 independent trials); for comparison purposes, we also report the average SINR obtained when the radars operate in a no-game scenario. The plots confirm that, at the Nash equilibria, the radar network actually may enjoy an increase in terms of SINR, with respect to the case in which no-game is allowed. Moreover, as expected, the performance gracefully degrades as the number of active radars increases. This is a pretty natural behavior, since the larger the number of radars the larger the power of the interfering signals.

Overall, the results of this section confirm the effectiveness of the proposed algorithms, as well as that all the considered games converge to an equilibrium.

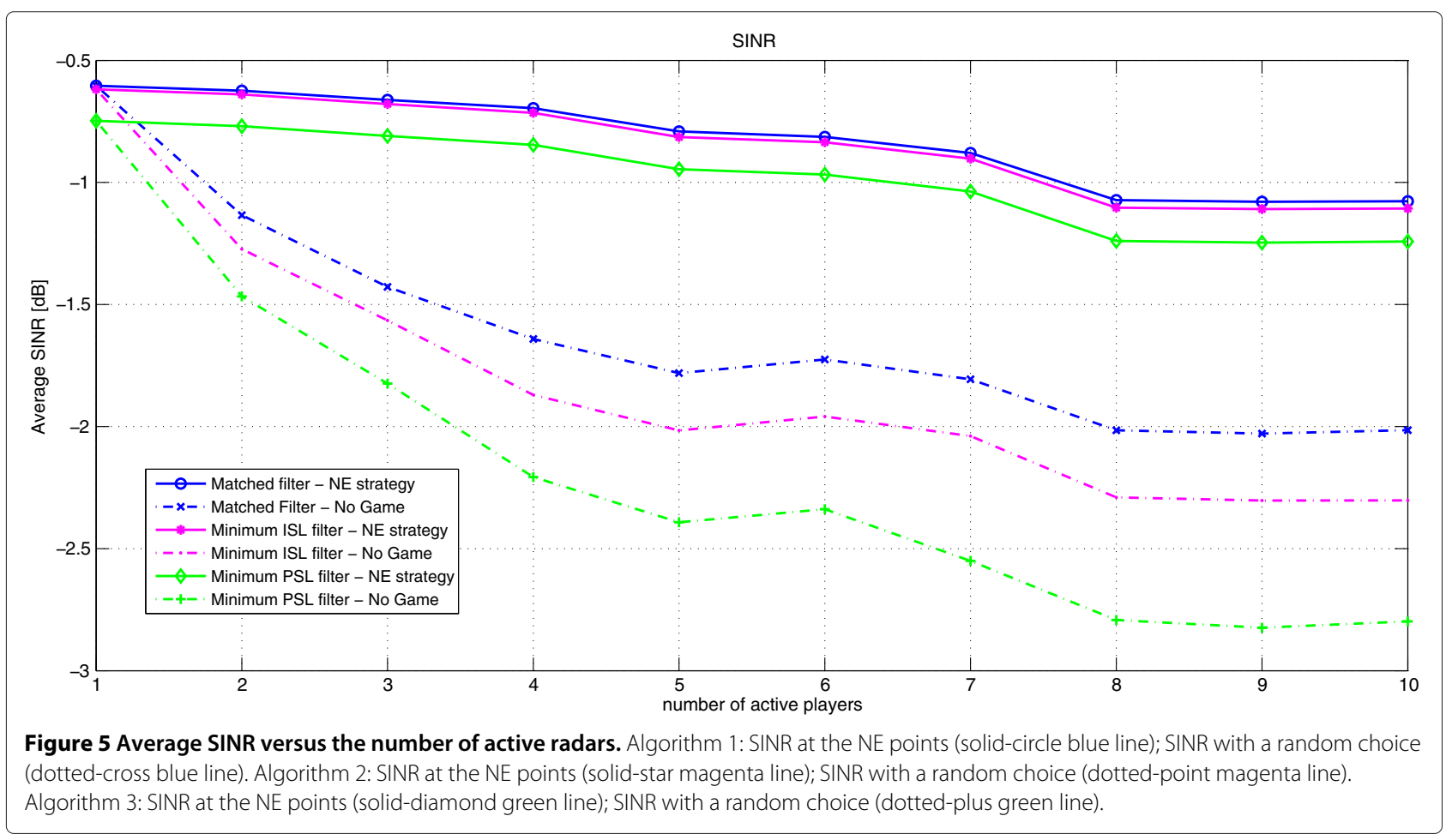




\section{Conclusion}

In this article, we have considered a network of radars sharing the same frequency band, and tuning their transmitted waveforms in order to improve their SINR.

We have assumed that each radar can select the waveform to be transmitted from a finite set. Hence, we have proposed code updating strategies according to some non-cooperative games, based on the potential games framework, to account for the cases of matched filter detection, minimum ISL and minimum PSL detection. Finally, we have discussed the situation where a nonnegligible Doppler shift exists in the received data. In all the considered scenarios, the existence of NE is analytically proven.

Numerical results have confirmed that the proposed games are effective in improving the system performance, in the sense that at the NE each radar may enjoy an SINR that is larger than that corresponding to the case of a random choice of the coded waveform to transmit. Moreover, it has also been verified that there is a graceful performance degradation as the number of active radars increases.

Possible future research tracks might account for the possibility of some form of cooperation between the radars of the network as well as the extension of the procedure to the case where more advanced decision strategies (in place of the linear filter followed by an envelope detector) are used. By doing so, we can also confer to the system additional desired robust features such as for instance the constant false-alarm rate property.

\section{Appendix}

\section{Code design procedure}

We choose our $N$-dimensional radar codes so that $\|\boldsymbol{c}\|=$ $1, c \in \mathbb{C}^{N}$; otherwise stated, we fill the set $\Omega$ with sequences lying on the unit-norm sphere. To this end, we consider both standard codes available in open literature and ad-hoc coding procedure.

As to the former class, we refer to some wellknown phase-coding techniques [35] to design the first 13 possible transmit sequences of the set $\Omega$. Specifically, we assume that $c_{l}=\frac{1}{\sqrt{N}} e^{\sqrt{-1} \phi_{l}}$, where $\phi_{l}=$ $\left[\phi_{l}(1), \ldots, \phi_{l}(N)\right]^{T}$ is the phase sequence of the $l$ th code, and $l=1, \ldots, 13$. In Table 1 , we summarize the classes of phase codes herein used, as well as the values of the parameters applied in the respective design procedures. ${ }^{\mathrm{e}}$

In addition, to properly test our non-cooperative procedures, we increase the number of possible strategies enriching with other suitable codes the set $\Omega$. We resort to the following construction procedure. First of all, we force the coefficients $c_{l}(i), i=1, \ldots, N$, to belong to a well-defined finite set $\Omega_{*}$ with cardinality $M$. Then, we obtain the transmit sequences picking up randomly the codes from the set $\Omega_{*}^{N}$ with cardinality $M^{N}$. Finally, we normalize the selected sequences so as to get unitnorm codes. For the specific case at hand, we set $c_{l}(i) \triangleq$ $\left\{a_{i}+\sqrt{-1} b_{i}\right\} / \sqrt{2 N}$ for $l=14, \ldots, 113$, with $\left\{a_{i}, b_{i}\right\} \in$ $\{-1,+1\}^{2}$. With such a choice we can produce up to $2^{2 N}$ possible codes. Thus, we randomly choose 100 codes from such a set, and use them in our simulations.

The aforementioned construction procedure does not provide sequences very attractive from the radar point of view; indeed, it can lead to signals with significant modulus variations, poor range resolution, high peak sidelobe levels, and more in general, to signals with an undesired ambiguity function behavior. These drawbacks can be circumvented imposing a control on the aforementioned performance metrics at the code design stage. Precisely, we can start from a good (in the sense of the ambiguity function properties) code $c_{0}$ and devise some additional sequences which inherit some attractive properties of $\boldsymbol{c}_{0}$. This goal can be achieved forcing the new sequences to lie in a suitable norm-ball centered around $\boldsymbol{c}_{0}$. In other words, we consider sequences which are solutions to the feasibility problem

$$
\left\{\begin{array}{l}
\left\|\boldsymbol{c}-\boldsymbol{c}_{\mathbf{0}}\right\|^{2} \leq \epsilon \\
\|\boldsymbol{c}\|^{2}=1
\end{array},\right.
$$

where the parameter $\epsilon \in[0,2]$ quantifies the desired similarity level; the smaller $\epsilon$, the higher the degree of similarity among the ambiguity functions of the designed radar code and the reference sequence.

Table 2 Set of similarity codes

\begin{tabular}{llll}
\hline Parameters & $\phi_{0}$ & Parameters & $\phi_{0}$ \\
\hline$r=3$ & Chu & - & Px \\
- & Frank & $r=17$ & Golomb-Zhang \\
- & MPS (Minimum Peak Sidelobe) & $r=6, q=6$ & Zadoff \\
- & P4 & - & Polyphase Barker \\
- & P1 & - & - \\
\hline
\end{tabular}

Set of similarity codes. $c_{0}=\frac{1}{\sqrt{N}} e^{j \phi_{0}}$ : reference code; length $N=16 ;\{r, q\}$ design parameters [35]. 
Solutions to problem (35) can be found according to the following algorithm.

1. Denote by $\boldsymbol{a}$ an $N$-dimensional complex vector whose elements are continuous random variables.

2. Construct the unit-norm vector $c_{0}^{\perp}=\left(I-c_{0} c_{0}^{\dagger}\right) a /\left\|\left(I-c_{0} c_{0}^{\dagger}\right) a\right\|$.

3. Define the sequence $\boldsymbol{c}^{t}=\sqrt{t} \boldsymbol{c}_{\mathbf{0}}+\sqrt{1-t} \boldsymbol{c}_{\mathbf{0}}{ }^{\perp}$, where the parameter $t$ complies with $t \geq(1-\epsilon / 2)^{2}=\delta_{\epsilon}$ and $t \leq 1$.

Exploiting the above procedure, we have updated the set $\Omega$, so as to include additional 540 transmit sequences. In Table 2, we show the set of reference codes; for each sequence, we solve problem (35) $K=15$ times (with 15 different feasible values of $t$ ), thus devising $9 K$ possible codes. Finally, the procedure is implemented for $\delta_{\epsilon} \in\{0.41,0.63,0.75,0.9\}$.

\section{Endnotes}

${ }^{a}$ Actually, the SINR definition should include also the coefficients $\alpha_{\text {., }}$; however, no prior knowledge of these coefficients may be reasonably assumed, and we are thus omitting them in the SINR definition reported in (4).

${ }^{\mathrm{b}} \mathrm{We}$ are considering a bi-dimensional scenario where $G(\theta)$ is the azimuth beam pattern. However, the extension to a three-dimensional situation accounting for both azimuth and elevation is quite easy.

${ }^{\mathrm{c}}$ With $\left|\Omega_{j}\right|$ we are denoting the cardinality of the set $\Omega_{j}$, whereas with $c_{j}^{i}$ we are indicating the $i$ th element of $\Omega_{j}$.

${ }^{\mathrm{d}}$ Recall that in the above gain matrices the $(m, n)$ th element is a coefficient weighting the interference from the $m$ th radar on the $n$th receiver.

${ }^{\text {e}}$ The reader might refer to [35], which is an exhaustive compendium of the classic radar coding techniques.

Competing interests

The authors declare that they have no competing interests.

\section{Acknowledgments}

The effort of A. De Maio and M. Piezzo is sponsored by the Air Force Office of Scientific Research, Air Force Material Command, USAF, under grant number FA8655-09-1-3006. The U.S. Government is authorized to reproduce and distribute reprints for Governmental purpose notwithstanding any copyright notation thereon.

\section{Author details}

${ }^{1}$ DIETI, University of Naples "Federico II", Napoli, Italy. ${ }^{2}$ CNR, IREA, via Diocleziano 328, I-80124, Napoli, Italy. ${ }^{3}$ DAEIMI, University of Cassino, Cassino (FR), Italy. ${ }^{4}$ SELEX Electronic Systems - A Finmeccanica Company, via Tiburtina Km.12.4, I-00131, Roma, Italy.

Received: 10 July 2012 Accepted: 28 February 2013

Published: 28 March 2013

\section{References}

1. CJ Baker, AL Hume, Netted radar sensing. IEEE Aerosp. Electron. Syst. Mag. 18(2), 3-6 (2003)
2. VS Chernyak, Fundamentals of Multisite Radar System: Multistatic Radars and Multiradar Systems. (Gordon and Breach Science Publishers, New York, 1998)

3. E Fishler, A Haimovich, R Blum, L Cimini, D Chizhik, R Valenzuela, Spatial diversity in radars: models and detection performance. IEEE Trans. Signal Process. 54(3), 823-838 (2006)

4. J Li, P Stoica, MIMO Radar Signal Processing. (Wiley, New York, 2008)

5. A De Maio, GA Fabrizio, A Farina, WL Melvin, L Timmoneri, in Proceedings of the IEEE International Radar Conference, 2007. Challenging issues in multichannel radar array processing (IEEE (Edinburgh, UK, April 2007), pp. 856-862

6. L Landi, R Adve, in Principles of Waveform Diversity and Design. Time-orthogonal-waveform-space-time adaptive processing for distributed aperture radars, Sect. B—part III, ch. 19 (SciTech Publishing Raleigh, NC, 2011)

7. RS Adve, RA Schneible, G Genello, P Antonik, in Proceedings of the IEEE International Radar Conference 2005. Waveform-space-time adaptive processing for distributed aperture radars (IEEE (Arlington, USA, May 2005), pp. 93-97

8. KH Berthke, B Röde, M Schneider, A Schroth, A novel non-cooperative near-range radar network for traffic guidance and control on airport surfaces. IEEE Trans. Control Syst. Technol. 1(3), 168-178 (1993)

9. H Huang, D Lang, in Proceedings of the IEEE International Radar Conference 2005. The comparison of attitude and antenna pointing design strategies of non-cooperative spaceborne bistatic radar (IEEE (Arlington, USA, May 2005), pp. 568-571

10. HD Ly, Q Liang, in Proceedings of the IEEE Military Communications Conference 2006. Spatial-temporal-frequency diversity in radar sensor networks (IEEE Washington, DC, USA, October 2006), pp. 1-7

11. N Levanon, Multifrequency complementary phase-coded radar signal. Proc. IEE Radar Sonar Navigat. 17(6), 276-284 (2000)

12. A Farina, in Proceedings of the Third International Waveform Diversity \& Design Conference 2007. Waveform diversity: past, present, and future (Plenary Talk Pisa, Italy, June 2007)

13. A Aubry, A De Maio, A Farina, M Wicks, Knowledge-aided (potentially cognitive) transmit signal and receive filter design in signal-dependent clutter. IEEE Trans. Aerosp. Electron. Syst. 49(1), 93-117 (2013)

14. A Aubry, A De Maio, M Piezzo, A Farina, M Wicks, Cognitive design of the receive filter and transmitted phase code in reverberating environment. IET Radar Sonar Navigat. 6(9), 822-833 (2012)

15. J Li, L Xu, P Stoica, KW Forsythe, DW Bliss, Range compression and waveform optimization for MIMO radar: a Cramér-Rao bound based study. IEEE Trans. Signal Process. 56(1), 218-232 (2008)

16. A De Maio, M Lops, Design principles of MIMO radar detectors. IEEE Trans. Aerosp. Electron. Syst. 43(3), 886-898 (2007)

17. A Aubry, M Lops, AM Tulino, L Venturino, On MIMO detection under non-gaussian target scattering. IEEE Trans. Inf. Theory. 56(11), 5822-5838 (2010)

18. N Subotic, K Cooper, P Zulch, in Proceedings of the International Waveform Diversity and Design Conference, 2007. Conditional and constrained joint optimization of radar waveforms (IEEE (Pisa, Italy, June 2007), pp. 387-394

19. M Greco, F Gini, P Stinco, A Farina, L Verrazzani, Adaptive waveform diversity for cross-channel interference mitigation (IEEE, (Roma, Italy, May 2008), pp. 1-6

20. G Scutari, DP Palomar, S Barbarossa, Competitive design of multiuser MIMO systems based on game theory: a unified view. IEEE J. Sel. Areas Commun. 26(7), 1089-1103 (2008)

21. A De Maio, F Gini, L Patton, in Waveform Design and Diversity for Advanced Radar Systems. Waveform design for non-cooperative radar networks, ch. 10 ((Institution of Engineering and Technology London, UK, 2012)

22. D Fudenberg, J Tirole, Game Theory. (MIT Press, Cambridge, MA, 1991)

23. D Monderer, LS Shapley, Potential games. Games Econ. Behav. 14(44), 124-143 (1996)

24. M Piezzo, S Buzzi, A De Maio, A Farina, in Evolutionary and Deterministic Methods for Design, Optimization and Control (EUROGEN). Non-cooperative code design in radar networks: a game-theoretic approach ((Capua, Italy, September 2011), pp. 14-16

25. S Buzzi, G Colavolpe, D Saturnino, A Zappone, Potential games for energyefficient power control and subcarrier allocation in uplink multicell OFDMA systems. IEEE J. Sel. Topics Signal Process. 6(2), 89-103 (2012) 
26. KR Griep, JA Ritcey, JJ Burlingame, Polyphase codes and optimal filters for multiple user ranging. IEEE Trans. Aereosp. Electron. Syst. 31(2), 752-767 (1995)

27. P Stoica, J Li, M Xue, Transmit codes and receive filters for radar: a look at the design process. IEEE Signal Process. Mag. 25(6), 94-109 (2008)

28. YI Abramovich, MB Sverdlik, Synthesis of a filter which maximizes the signal-to-noise ratio under additional quadratic constraints. Radio Eng. Electron. Phys. 15(11), 1977-1984 (1970)

29. YI Abramovich, MB Sverdlik, Synthesis of filters maximizing the signal-tonoise ratio in the case of a minimax constraint on the sidelobes of the crossambiguity function. Radio Eng. Electron. Phys. 16, 253-258 (1971)

30. S Zoraster, Minimum peak range sidelobe filters for binary phase-coded waveforms. IEEE Trans. Aereosp. Electron. Syst. 16(1), 112-115 (1980)

31. JC Liberti Jr, TS Rappaport, Analytical results for capacity improvements in CDMA. IEEE Trans. Veh. Technol. 43(3), 680-690 (1994)

32. S Anderson, M Millnert, M Viberg, B Wahlberg, An adaptive array for mobile communication systems. IEEE Trans. Veh. Technol. 40(1), 230-236 (1991)

33. VT Dolgochub, MB Sverdlik, Generalized $\gamma$-filters. Radio Eng. Electron. Phys. 15, 147-150 (1970)

34. P Stoica, J Li, M Xue, On binary probing signals and instrumental variables receivers for radar. IEEE Trans. Inf. Theory. 54(8), 3820-3825 (2008)

35. N Levanon, E Mozeson, Radar Signals. (Wiley, New York, 2004)

\section{doi:10.1186/1687-6180-2013-63}

Cite this article as: Piezzo et al:: Non-cooperative code design in radar networks: a game-theoretic approach. EURASIP Journal on Advances in Signal Processing 2013 2013:63.

\section{Submit your manuscript to a SpringerOpen ${ }^{\odot}$ journal and benefit from:}

- Convenient online submission

- Rigorous peer review

- Immediate publication on acceptance

- Open access: articles freely available online

- High visibility within the field

- Retaining the copyright to your article

Submit your next manuscript at $\boldsymbol{\triangleright}$ springeropen.com 